\title{
73793 Biofiltration of Volatile Pollutants: Solubility Effects
}

\author{
Publication Date: June 15, 2001
}

Principal Investigator: Dr. Brian H. Davison, Oak Ridge National Laboratory, PO Box 2008, MS 6226, Oak Ridge, TN 37831, (865) 576-8522; davisonbh@ornl.gov

Co-Investigator: Dr. John W. Barton, (865) 241-5706, bartonjw@ornl.gov

Graduate Students: Two BS candidates will complete student internships for this project during the second year.

\section{RESEARCH OBJECTIVE}

This project investigates and collects fundamental partitioning data for a variety of sparingly soluble subsurface contaminants (e.g., TCE, etc.) between vapor, aqueous phase, and matrices containing substantial quantities of biomass and biomass components. Due to the difficulty of obtaining these measurements, environmental models have generally used solubility constants of chemicals in pure water or, in a few rare cases, simple linear models. Our prior EMSP work has shown that the presence of biological material can increase effective solubilities by an order of magnitude for sparingly soluble organics; therefore, the previous simple approaches are not valid and are extremely poor predictors of actual bio-influenced partitioning. It is likely that environmental contaminants will partition in a similar manner into high-biomass phases (e.g. biobarriers and plants) or humic soils. Biological material in the subsurface can include lipids, fatty acids, humic materials, as well as the lumped and difficult-to-estimate 'biomass'. Our measurements include partition into these biological materials to allow better estimation. Fundamental data collected will be used in mathematical models predicting transport and sorption in subsurface environments, with the impacts on bioremediation being evaluated based on this new information. Our 2-D Win95/98/XP software program, Biofilter 1.0, developed as a part of our prior EMSP efforts for describing biofiltration processes with consideration given to both kinetic and mass transfer factors, is being extended to incorporate and use this information.

\section{RESEARCH PROGRESS AND IMPLICATIONS}

This report summarizes work after 18 months of a 3-year project. Experiments have been initiated which focus on benzene, toluene, trichloroethylene (TCE), and methyl chloride (MC) as critical contaminants of interest. We have developed a number of new protocols for precise measurements for complex multiphase systems containing different types of biomass, octanol and other organic liquids, water, and a headspace. Some of the key results have been:

1. Greater-than-expected biomass partitioning into organic phases such as octanol. 'Equilibrium' mixtures have indicated that as much as $1 \%$ of aqueous-phase biomass--in the form of whole yeast cells--can permeate an adjacent octanol phase, which is much higher than intuitively obvious.

2. Different types of biomass impact solubility of target contaminants to varying extents. Simple agar, for example, had minimal impact (no statistically significant impact within experimental error) on the solubility of several sparingly soluble organics. On the other hand, the presence of whole-yeast cells increased effective aqueous TCE solubility by more than 2 orders of magnitude.

3. Partitioning constants were found to be up to $33 X$ for BTEX constituents in the presence of biomass as compared to those for pure water. Benzene and toluene both exhibit dramatic changes in their Henry's Law partitioning values in the presence of biomass. 
4. We have begun examination of individual cell/biomass component contributions, using 'pure' biomasses such as albumin (protein), soluble starch and cellulose (complex carbohydrates), and lipids. These results could make it possible to predict, a priori, solubility enhancement based on chemical characterization of a particular environment and/or cell/biomass. As far as we are aware, correlations of this nature have not been previously attempted or reported.

Implications. This research will provide much-needed fundamental information regarding partitioning of priority contaminants in subsurface aquifers, vadose zones, and surface water impoundments where biological material is present. Due to the difficulty of obtaining these measurements, environmental models have generally used solubility constants of chemicals in pure water or, in a few rare cases, simple linear models. Our work (see Figures 1 and 2) has shown that the presence of biological material can increase effective solubilities by more than an order of magnitude; therefore, the previous simple approaches are not valid and are extremely poor predictors of actual bio-influenced partitioning. Accurate data measurements of phase distributions of sparingly soluble organics between biomaterial/water/air are needed to allow these data to be easily utilized by developers of predictive models for subsurface transport and fate as well as bioremediation. Figure $\mathbf{2}$ is particularly striking, because it shows that TCE solubility can change by more than two orders of magnitude over a short range of biomass addition. TCE is the nation's largest groundwater contaminant.

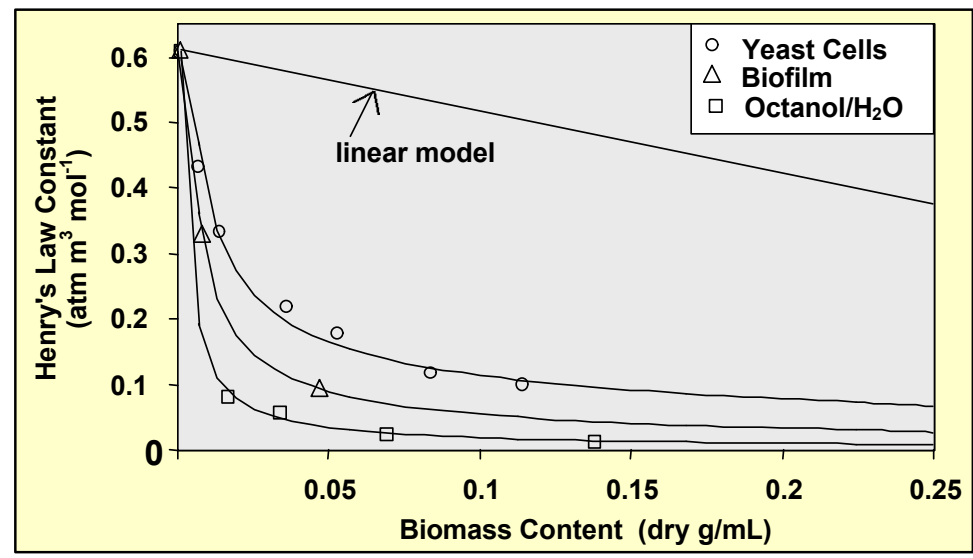

Figure 1. Published data for propane which show deviation from ideal models of partitioning between biomass and gas phases (as would be likely in vadose zones and surface impoundments). Data were collected for yeast (circles) and bacteria (triangles) over a variety of organic densities and compared to two 'ideal models' for octanol/water partitioning (squares and straight line). In both cases, actual partitioning measurements deviated significantly from idealized models for pure water/octanol mixtures.

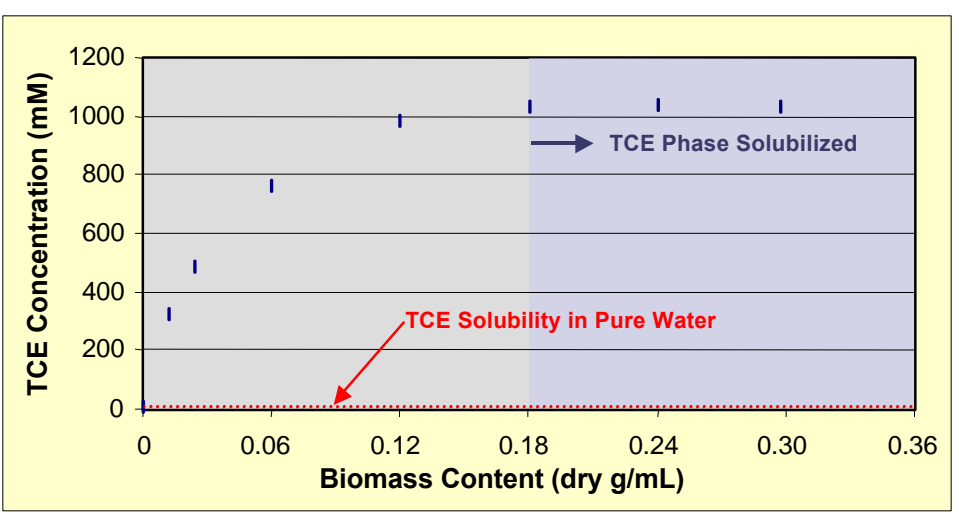

Figure 2. An aqueous phase containing varying amounts of whole cell biomass (in the form of yeast) was contacted with a constant volume of TCE; the volume ratio was 13:1. As the biomass concentration was raised, the TCE phase gradually disappeared until only one phase was present, which then had a constant TCE concentration. TCE in all phases was measured and accounted for, allowing for closure of the TCE mass balance.

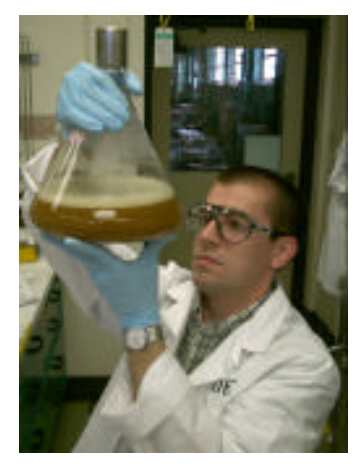

Biomass is harvested (see picture at left) from reactor vessels ranging in size from 4 to $500 \mathrm{~L}$ for use in partitioning experiments. Tightly sealed equilibrium test reactors (see picture at right) are constructed with precisely measured amounts of water, biomass, organic carrier, and target contaminant (such as TCE). Fundamental partitioning data are then incorporated into a standardized model for comparison and impact. Biomass types being investigated include yeast, bacteria, and traditional groupings of protein, lipid, and complex carbohydrates.

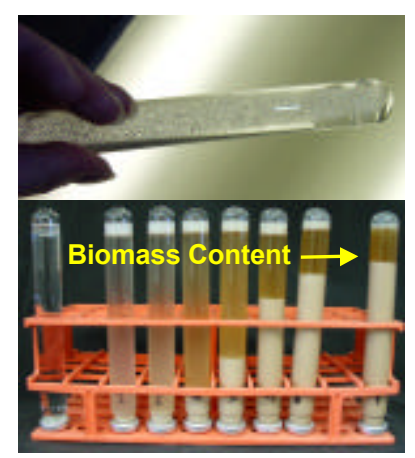




\section{PLANNED ACTIVITIES}

Experiments are being conducted at bench-scale to determine the physical partitioning parameters (equilibrium aqueous/biomaterial partition constants and Henry's law constants) of priority contaminants in contact with high biological material levels. Laboratory-scale flow-columns with porous matrices will be used to simulate subsurface aquifer conditions, allowing validation of models and impact of measured parameters. The following time/table lists/describes activities:

\section{Tasks/Time Table}

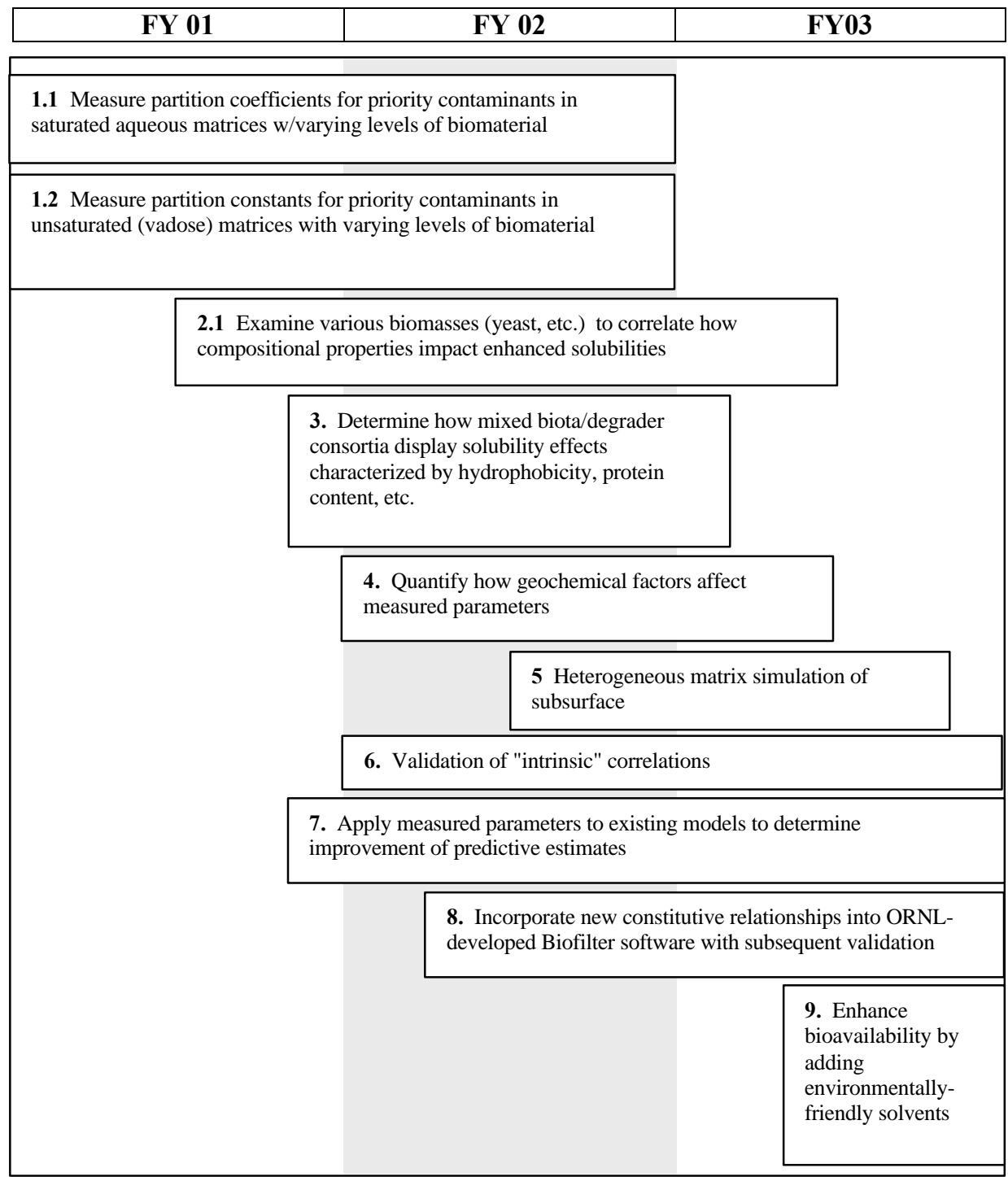

\section{INFORMATION ACCESS}

Barton, JW, SA Jones, CD Vodraska, BH Davison. The Effect of Biomass on the Measured Solubility of Priority Contaminants in Aqueous Bioremediation Systems. In preparation, 2002.

Barton, JW, GS Shinu, CD Vodraska, BH Davison. The Effect of Biomass on the Measured Solubility of Priority Contaminants in Aqueous Bioremediation Systems. Presentation at the American Institute of Chemical Engineers (AICHE) National Meeting, November 4-9, 2001, Reno, Nevada.

Davison, B.H., J.W. Barton, K.T. Klasson, A. F. Francisco. "Influence of Biomass on Alkane Solubilities". Biotechnology and Bioengineering, 68:279-284, 2000.

Davison, BH, Barton, JW, Klasson, KT, Francisco AB. Effect of biomass on the measured solubility of sparingly soluble organics in aqueous bioremediation systems. Presented at the $217^{\text {th }}$ ACS National Meeting, March 21-25, 1999, Anaheim, CA.

Biofilter Program, Beta version - available for download at www.ct.ornl.gov/biofilter 